\title{
Neutrophils isolated from the synovial fluid of patients with rheumatoid arthritis: priming and activation in vivo
}

\author{
Heather L Nurcombe, Roger C Bucknall, Steven W Edwards
}

\begin{abstract}
The oxidative metabolism of neutrophils isolated from the bloodstream and synovial fluid of 16 patients with rheumatoid arthritis was compared by measuring the ability of neutrophils to generate luminol dependent chemiluminescence and to secrete $\mathrm{O}_{2}^{-}$. Measurements of receptor mediated-that is, $\boldsymbol{N}$-formylmethionyl-leucyl-phenylalanine stimulatedactivation, or receptor and second message independent-that is phorbol myristate acetate stimulated-activation showed that synovial fluid neutrophils had biochemical characteristics to suggest that they had been either up-regulated (primed) or down-regulated (activated) in vivo. These conclusions were confirmed by comparison of these responses with the changes in oxidative metabolism observed during in vitro priming and activation of control neutrophils: synovial fluid neutrophils possessed lower levels of myeloperoxidase than paired bloodstream cells, and unlike bloodstream cells could not be primed in vitro. These data thus suggest that synovial fluid neutrophils have been exposed to both priming and activating agents within rheumatoid joints.
\end{abstract}

Phagocytic cells of the immune system, such as polymorphonuclear leucocytes and macrophages, can be stimulated under appropriate conditions to secrete a series of reactive oxygen metabolites and a range of proteolytic enzymes. ${ }^{1}$ Because these cells are present in large numbers in the synovial fluid of patients with rheumatoid arthritis it has been proposed that inappropriate release of oxidants and proteolytic enzymes from activated neutrophils is responsible, at least in part, for the joint damage associated with this disease. ${ }^{2-4}$ Indeed, in vitro, reactive oxidants can degrade/depolymerise the hyaluronic acid of synovial fluid thereby reducing its viscosity, attack cartilage and other tissues, and also mmediate a number of other proinflammatory and anti-inflammatory events. ${ }^{2}$ 4-7 Immune complexes which are present within these joints seem to be likely candidates as neutrophil activating substances. 89

Several lines of evidence are now emerging to confirm the hypothesis that synovial fluid neutrophils are indeed activated in situ within rheumatoid joints. Although it is not possible to measure levels of oxidants directly (because of their high chemical reactivity), oxidatively damaged products have been detected within rheumatoid joints. ${ }^{10} 11$ Similarly, neutrophils isolated from the synovial fluid of patients with rheumatoid arthritis have surface receptor expression characteristic of activated cells, ${ }^{12} 13$ and myeloperoxidase (a neutrophil protein capable of producing $\mathrm{HOCl}^{14}$ ) is present in synovial fluid in a form which suggests that it has been cosecreted with oxidants. ${ }^{15}$ Furthermore, analysis of the oxidative metabolism of paired bloodstream and synovial fluid neutrophils suggests that the latter cells have been activated within the joint. ${ }^{16} 17$

It is now recognised that in addition to their ability to become activated in response to inflammatory stimuli, bloodstream neutrophils can also be 'primed' by suboptimal concentrations of stimuli ${ }^{18}$ or upon exposure to cytokines ${ }^{19}$ into a state of enhanced functional responsiveness. 'Priming' in vivo or in vitro results in molecular modifications to the neutrophil, which can be detected by suitable assays, such as determination of expression of surface receptors or responsiveness to receptor mediated stimulation. As cytokine production has been implicated in the pathology of rheumatoid arthritis, ${ }^{20-27}$ the aim of this work was to establish whether neutrophils attracted from the bloodstream into rheumatoid joints express the characteristics of 'primed' or activated cells, or both.

\section{Patients and methods}

PATIENTS

Sixteen patients attending the rheumatology clinics of the Royal Liverpool Hospital, all with classical or definite seropositive rheumatoid arthritis (American Rheumatism Association criteria), were studied. Their mean age was 56 years (range 24-80) and disease duration 8.8 years (range $0 \cdot 3-31$ ). All were receiving nonsteroidal anti-inflammatory drugs and no patient had received steroids within the previous six months. Control blood was taken from healthy laboratory personnel.

\section{PREPARATION OF NEUTROPHILS}

Neutrophils were prepared from $20 \mathrm{ml}$ heparinised venous blood using M-PRM (Flow Laboratories) exactly as described previously, ${ }^{28}$ and from synovial fluid as described in ref 15 . After purification they were suspended in a buffer containing (mM): $\mathrm{NaCl} 120 ; \mathrm{KCl} \mathrm{4.8}$; $\mathrm{KH}_{2} \mathrm{PO}_{4} 1 \cdot 2 ; \mathrm{CaCl}_{2} 1 \cdot 3 ; \mathrm{MgSO}_{4} 1 \cdot 2 ; \operatorname{HEPES}(N$ 2-hydroxyethylpiperazine- $N^{\prime}$-2-ethanesulphonic acid) 25 (pH 7.4); 0.1\% bovine serum albumin. Cells were counted after a suitable dilution in the above buffer using a Fuchs-Rosenthal haemocytometer slide and used within four hours of preparation. 
ANALYTICAL METHODS

Neutrophils were suspended in buffer containing $10 \mu \mathrm{M}$ luminol (5-amino-2,3-dihydro-1,4phthalazenedione) at $10^{6}$ cells $/ \mathrm{ml}$ in a total volume of $1 \mathrm{ml},{ }^{29}$ and chemiluminescence was measured at $37^{\circ} \mathrm{C}$ with an LKB Wallac 1250 or 1251 luminometer.

$\mathrm{O}_{2}^{-}$generation was measured in a continuous assay by monitoring the rate of superoxide dismutase inhibitable reduction of ferricytochrome $c .^{30}$ The assay, total volume $1 \mathrm{ml}$, was performed in a Perkin-Elmer Lambda 5 spectrophotometer and contained $75 \mu \mathrm{M}$ cytochrome $c$ plus $5 \times 10^{5}$ cells.

Myeloperoxidase activity was measured by the rate of formation of tetraguaiacol from guaiacol. $^{31}$

\section{Results}

OXIDATIVE METABOLISM OF NEUTROPHILS ISOLATED FROM PATIENTS WITH RHEUMATOID ARTHRITIS

Neutrophils were isolated from the synovial fluid of patients with rheumatoid arthritis and the ability of these cells to generate reactive oxidants was compared with that of paired bloodstream cells isolated from the same patients. Thus this approach directly measured the change in functional activity of these cells after they had migrated from the bloodstream into the inflamed joint. Oxidant generating ability was assessed by luminol dependent chemiluminescence, which measures both intracellular and extracellular activity of the myelo-
A

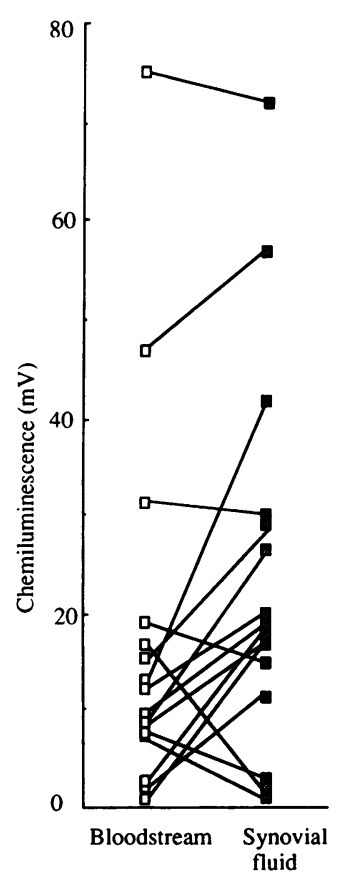

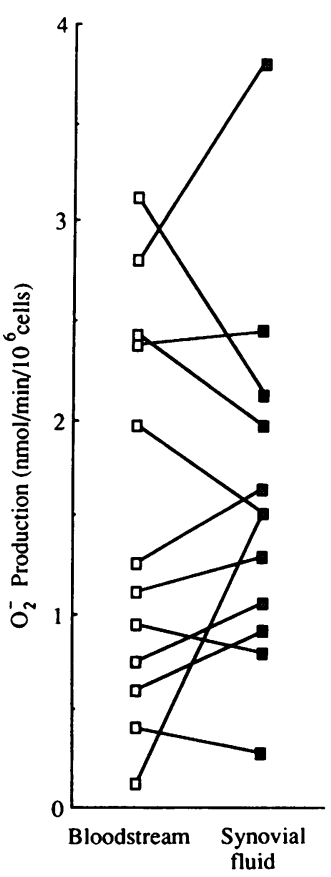

Figure 1 fMet-Leu-Phe stimulated oxidant generation. Neutrophils were isolated from the bloodstream and synovial fluid of patients with rheumatoid arthritis, as described in 'Patients and methods'. (A) Neutrophils $\left(10^{6} \mathrm{cells} / \mathrm{ml}\right)$ were suspended in buffer containing $10 \mathrm{uM}$ luminol; $(B)$ cells suspended in buffer containing $10 \mu M$ luminol; (B) cells $\left(5 \times 10^{5} / \mathrm{ml}\right)$ were suspended in buffer containing $75 \mu M$
cytochrome c. Stimulation was achieved by the addition of $1 \mu M$ fMet-Leu-Phe, and maximal rates of

chemiluminescence or cytochrome $c$ reduction were measured. peroxidase- $\mathrm{H}_{2} \mathrm{O}_{2}$ system, ${ }^{29} 32$ and by cytochrome $c$ reduction, which measures the rate of $\mathrm{O}_{2}^{-}$secretion. The stimulants used (at maximal activating doses) were $N$-formyl-methionylleucyl-phenylalanine(fMet-Leu-Phe; to measure receptor mediated activation), fMet-Leu-Phe plus cytochalasin $B$ (to assess the ability of these cells to degranulate), and phorbol myristate acetate (PMA), which activates these cells independently of plasma membrane receptor occupancy and second messenger generation.

\section{fMet-Leu-Phe}

The ability of neutrophils isolated from the bloodstream of patients with rheumatoid arthritis to generate luminol dependent chemiluminescence in response to fMet-LeuPhe showed considerable variation (fig 1A). Thus maximal rates of chemiluminescence varied over a 50 -fold range, which was a much greater variation in response than that noted in bloodstream neutrophils isolated from healthy controls (unpublished observations). Similarly, the range in fMet-Leu-Phe responsiveness of neutrophils isolated from the synovial fluid of these patients varied considerably. Comparison of the chemiluminescence responses of paired samples showed that in $6 / 16$ patients $(38 \%)$ the synovial fluid neutrophils had a lower response than that noted in bloodstream cells, whereas in $10 / 16$ patients $(63 \%)$ the synovial fluid neutrophils had a greater responsiveness to this stimulant. When $\mathrm{O}_{2}^{-}$secretion in response to fMet-Leu-Phe was measured it was found that in $5 / 12$ patients $(42 \%)$ the synovial fluid neutrophils had lower responses than the paired bloodstream cells (fig 1B).

\section{Phorbol myristate acetate}

The ability of neutrophils to generate oxidants in response to receptor mediated activation depends largely upon their level of expression of membrane receptors and the ability of the NADPH oxidase to become activated. Both of these factors are dependent upon the past history of the cells (see later). One way of bypassing receptor mediated activation and subsequent generation of second messengers is to use PMA, which activates the oxidase by stimulating protein kinase $\mathrm{C}$ directly.

Phorbol myristate acetate was therefore used to stimulate luminol dependent chemiluminescence (fig $2 \mathrm{~A}$ ) and $\mathrm{O}_{2}^{-}$secretion in bloodstream and synovial fluid neutrophils. Again, considerable variations in maximal rates of oxidant generation were noted, but in all samples analysed (14) the ability of synovial fluid neutrophils to generate oxidants in response to this stimulant was less than in paired bloodstream cells.

\section{fMet-Leu-Phe plus cytochalasin B, and} myeloperoxidase secretion

The inhibitor of microfilament assembly, cytochalasin B, affects neutrophil responsiveness to fMet-Leu-Phe in several ways. Firstly, the rate of oxidant production is increased three to four- 

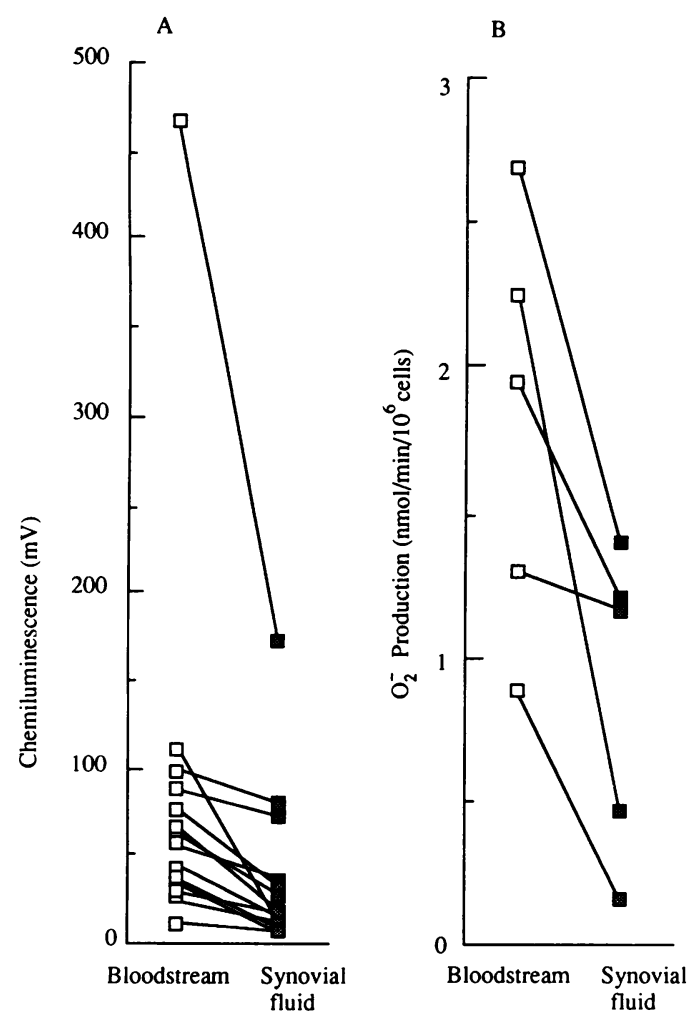

Figure 2 Phorbol myristate acetate (PMA) stimulated oxidant production. Experimental conditions were exactly as described in the legend to fig 1 except that stimulation was achieved by the addition of $0 \cdot 1 \mu \mathrm{g} / \mathrm{ml} P M A$.
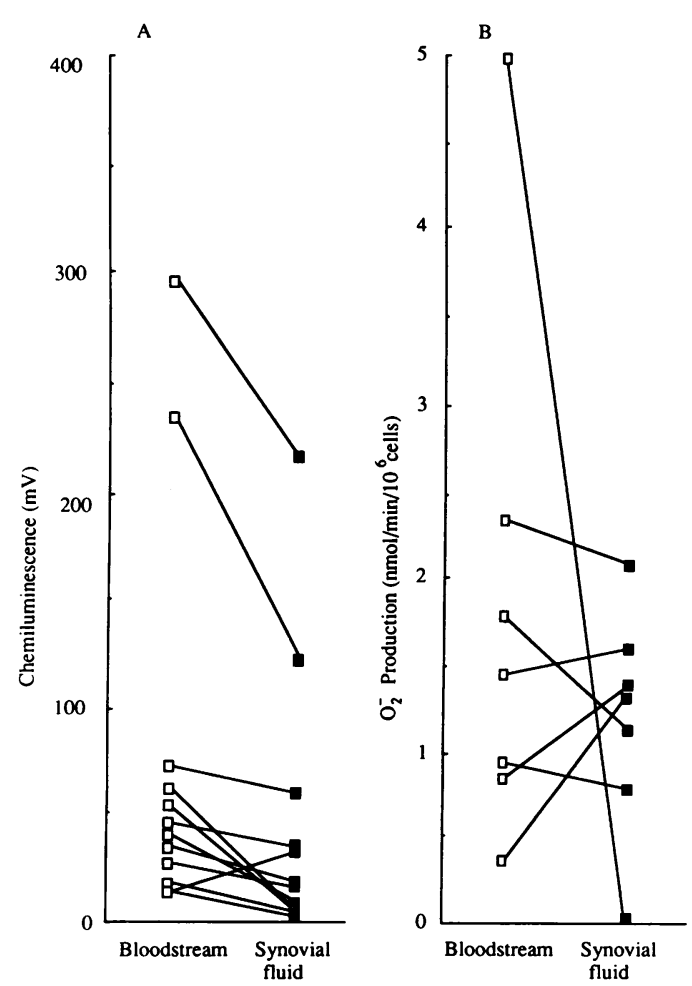

Figure 3 fMet-Leu-Phe plus cytochalasin B stimulated oxidant generation. Experimental conditions were exactly as described in the legend to fig 1 except that stimulation was achieved by the addition $1 \mu \mathrm{M}$ fMet-Leu-Phe plus $5 \mu \mathrm{g} / \mathrm{ml}$ cytochalasin B (final concentrations). fold $^{33}$; secondly, there is a rapid and extensive secretion of myeloperoxidase and other granule enzymes $^{34}$; and thirdly, this extracellular myeloperoxidase results in a greatly enhanced luminol dependent chemiluminescence as this assay is largely dependent upon the activity of the myeloperoxidase $-\mathrm{H}_{2} \mathrm{O}_{2}$ system. ${ }^{29}$

When oxidant generation was measured after stimulation by fMet-Leu-Phe plus cytochalasin $B$, the response of the synovial fluid neutrophils was reduced in $11 / 12$ patient samples (92\%) compared with paired bloodstream cells (fig 3A): the mean (SD) response of the synovial fluid cells was only 48 (22)\% of that of the paired bloodstream cells. Measurements of $\mathrm{O}_{2}^{-}$ were more varied as only four of the seven samples showed a similarly reduced response in the synovial fluid, the other three showing greater activity.

As luminol dependent chemiluminescence (unlike $\mathrm{O}_{2}^{-}$secretion) is largely dependent upon the activity of the myeloperoxidase $-\mathrm{H}_{2} \mathrm{O}_{2}$ system we measured the levels of activity of this enzyme in paired synovial fluid and bloodstream neutrophils. In all the patients (13) the activity of this enzyme in the synovial fluid cells was greatly reduced (fig 4), indicating that it had

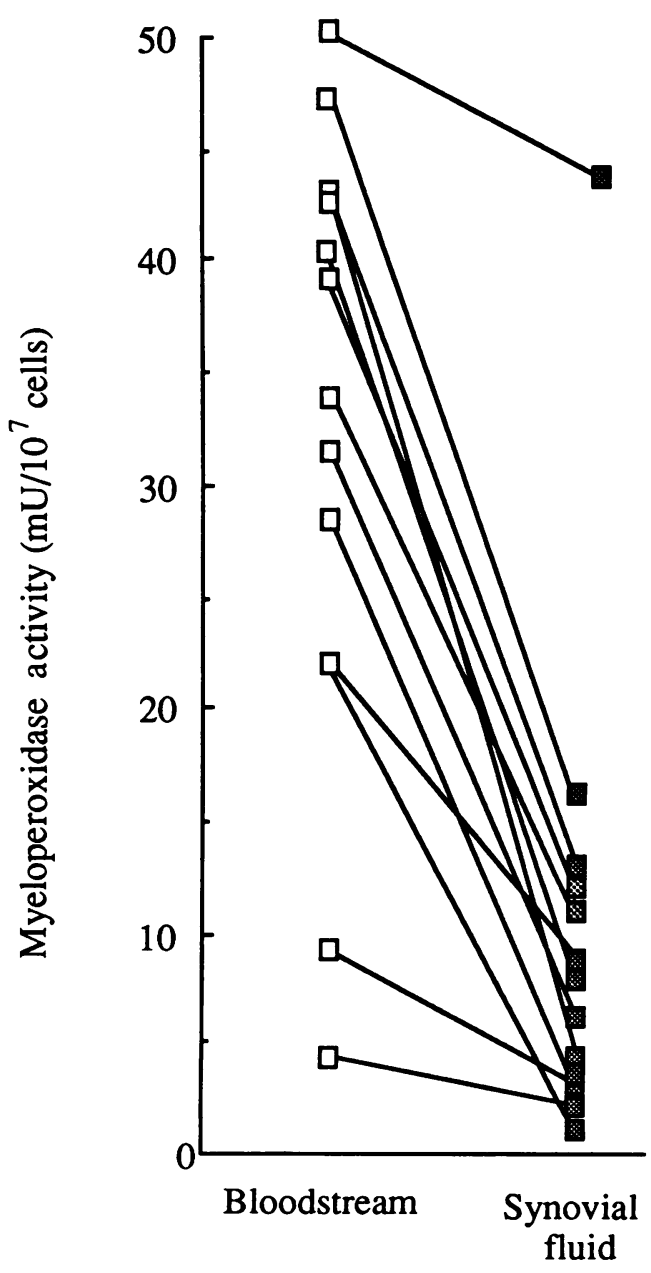

Figure 4 Myeloperoxidase activity of bloodstream and synovial fluid neutrophils. Neutrophils were isolated from the bloodstream and synovial fluid of patients with rheumatoid arthritis. Cells were lysed by the addition of $0.5 \%(v / v$, final concentration) Triton X-100 and two cycles of freeze/ thäwing. Myeloperoxidase activity was then measured by the guaiacol method. 


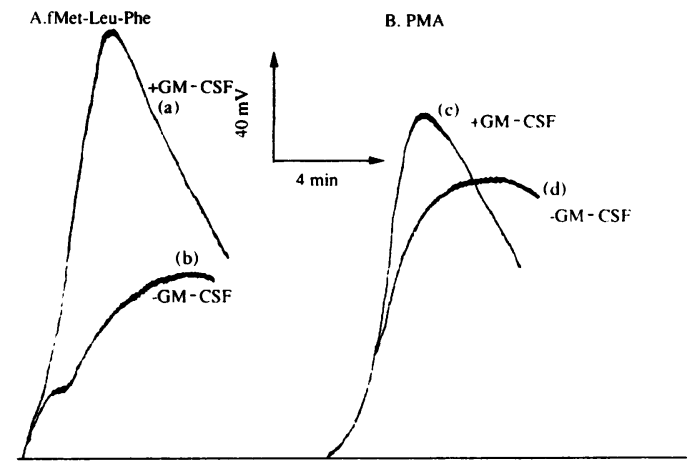

Figure 5 Effect of granulocyte-macrophage colony stimulating factor $(G M-C S F)$ on stimulated oxidant production. Neutrophils were isolated from the bloodstream of healthy volunteers and suspended in buffer at $10^{6} \mathrm{cells} / \mathrm{m} l$ in the presence $(a, c)$ or absence $(b, d)$ of $50 \mathrm{U} / \mathrm{ml}$ GM-CSF ${ }^{36}$ After one hour's incubation at $37^{\circ} \mathrm{C}$ luminol was added to $10 \mu M$ (final concentration) and

chemiluminescence was measured after the addition of $I \mu M$ $f M e t-L e u-P h e(a, b)$ or $0.1 \mu \mathrm{g} / \mathrm{ml}$ phorbol myristate acetate $(P M A)(c, d)$. Chemiluminescence traces were obtained with an LKB 1251 luminometer.

been secreted from the cells within the rheumatoid joint, confirming our earlier observations. ${ }^{15}$ Hence the greatly reduced response of the synovial fluid neutrophils to fMet-Leu-Phe plus cytochalasin B is explained by the greatly reduced levels of myeloperoxidase in these cells.

EFFECT OF PRETREATMENT WITH PRIMING AND STIMULATING AGENTS ON OXIDANT GENERATION The ability of neutrophils to generate reactive oxidants is dependent upon their past history. For example, if they have previously been exposed to priming agents, such as interferon gamma and granulocyte-macrophage colony stimulating factor (GM-CSF), their ability to generate oxidants in response to stimulation by fMet-Leu-Phe is greatly enhanced. ${ }^{35}{ }^{36}$ Figure 5A shows that when bloodstream neutrophils were exposed to GM-CSF for one hour before addition of fMet-Leu-Phe their ability to generate luminol dependent chemiluminescence was potentiated $2 \cdot 5$-fold. When GM-CSF primed neutrophils were stimulated with PMA (which bypasses membrane receptors and second message generation) only a marginal (up to $20 \%$ ) potentiation of the response was observed (fig 5B). This result is explained by the fact that the major mechanism by which priming agents up-regulate oxidative metabolism is through up-regulation of certain plasma membrane receptors. ${ }^{19}$

The ability of synovial fluid and bloodstream neutrophils to respond to the priming agent interferon gamma was therefore investigated. Figure 6 shows an experiment typical of 10 others. Although bloodstream neutrophils from patients with rheumatoid arthritis could be primed into a state of enhanced responsiveness by interferon gamma, synovial fluid neutrophils could not be similarly up-regulated. Thus bloodstream neutrophils showed a two to threefold increased oxidant generating ability in response to fMet-Leu-Phe, whereas the corresponding synovial fluid neutrophils showed a

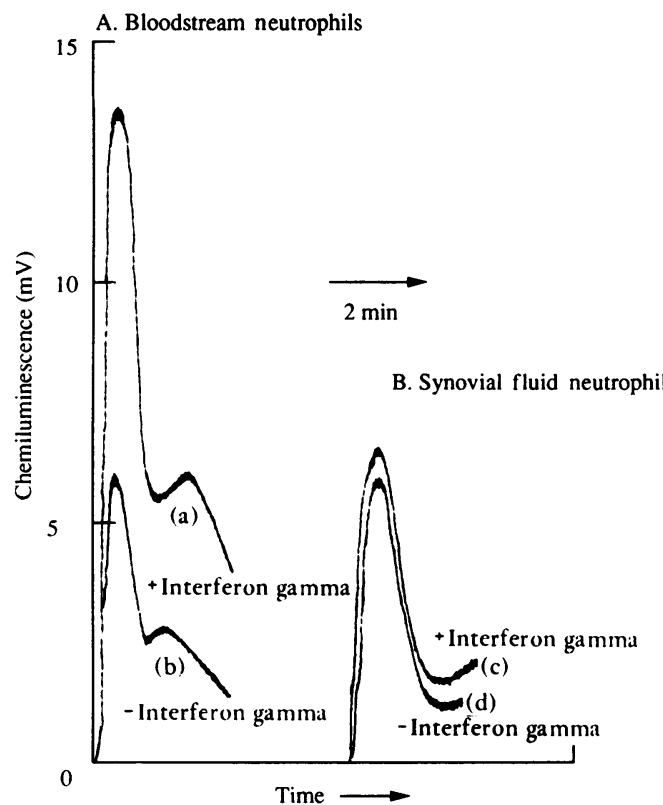

Figure 6 Effect of interferon gamma on bloodstream and synovial fluid neutrophil oxidant production. Neutrophils were isolated from the bloodstream $(a, b)$ and synovial fluid $(c, d)$ of patients with rheumatoid arthritis and suspended at $37^{\circ} \mathrm{C}$ for one hour in the presence $(a, c)$ and absence $(b, d)$ of $37^{\circ} \mathrm{C}$ for one hour in the presence $(a, c)$ and absence $(b, d)$ of
$100 \mathrm{U} / \mathrm{ml}$ interferon gamma. ${ }^{35}$ Luminol was added to $10 \mu \mathrm{M}$ (final concentration) and chemiluminescence measured after the addition of $1 \mu \mathrm{M}$ fMet-Leu-Phe (final concentration). Similar results were obtained with 10 other paired patient samples. Chemiluminescence traces were obtained with an $L K B 1250$ luminometer.

lower enhancement $(0-20 \%)$. Thus these data suggest that synovial fluid neutrophils cannot respond to priming agents in vitro, perhaps because they have already been exposed to such agents in vivo. As priming agents only upregulate responsiveness to certain membrane receptors, these data also suggest that the enhanced receptor mediated oxidant generation noted for some synovial fluid neutrophils (figs 1 and 3) may be due to the fact that these cells have previously been primed in vivo within the joints.

We then investigated the effects of previous stimulation of neutrophils on their responsiveness to subsequent activation by PMA. Thus bloodstream neutrophils were exposed to different concentrations of $\mathrm{fMet}$-Leu-Phe for 45 minutes before addition of PMA and measurement of luminol dependent chemiluminescence (fig 7). Cells pretreated with 1.0 or $0.5 \mu \mathrm{M}$ fMet-Leu-Phe could not be stimulated to generate luminol dependent chemiluminescence in response to PMA, but after pre-exposure to $0.2 \mu \mathrm{M}$ fMet-Leu-Phe (which resulted in $15 \%$ of the activity stimulated by saturable concentrations of this agent), a subsequent response to PMA was noted, but this was only $50 \%$ of that found in the absence of fMet-Leu-Phe pretreatment.

Hence prior suboptimal stimulation of neutrophils down-regulates their ability to generate oxidants in response to PMA. Therefore, these observations suggest that the reduced responsiveness of synovial fluid neutrophils to PMA (seen in fig 2) is due to the fact that these cells have previously been exposed to 


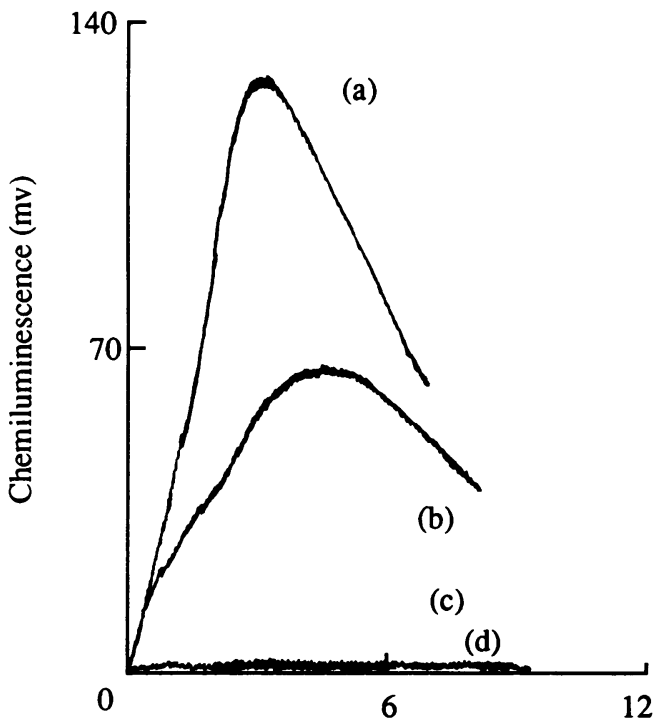

Time (min)

Figure 7 Effect of $f M e t-L e u-P h e$ pretreatment on phorbol myristate acetate (PMA) stimulated oxidant production. Neutrophils were isolated from the bloodstream of healthy volunteers and suspended in buffer containing $10 \mu M$ luminol and $f M e t-L e u-P h e$ at concentrations of $0(a), 0.2(b), 0.5(c)$, and $1 \cdot 0(d) \mu M$. After incubation at $37^{\circ} \mathrm{C}$ for 30 minutes $P M A$ was added (to $0.1 \mu \mathrm{g} / \mathrm{ml})$ and the resulting chemiluminescence measured with an LKB 1251 luminometer.

activating factors in vivo within rheumatoid joints.

\section{Discussion}

The ability of neutrophils to generate reactive oxidants is an important facet in their armoury to protect the host against invading microbial pathogens. Because these oxidants indiscriminately attack all biological molecules their production is carefully regulated, and during phagocytosis is restricted to within the phagolysosome. Activation of reactive oxidant production is a complex process and largely regulated in vivo by specific receptor/agonist interactions at the plasma membrane:oxidase activation during phagocytosis is mainly facilitated through Fc-receptor and complementreceptor occupancy during adherence of the phagocytes to opsonised bacteria. Thus immune complexes of immunoglobulins and complement fragments may inappropriately activate reactive oxidant production by neutrophils, and it has been proposed that such immune complexes, which are detectable in inflammatory diseases, are instrumental in activating neutrophils within rheumatoid joints. It is thus of vital importance that the role of neutrophil derived oxidants in the pathology of inflammatory joint disease is clearly defined.

Because of their high chemical reactivity it is not possible directly to measure released neutrophil derived oxidants within synovial fluid. Our approach used in this study was to identify the past history of synovial fluid neutrophils by measuring their responsiveness to specific exogenously added components: these responses were then directly compared with those obtained from bloodstream neutrophils from the same patients and with control neutrophils activated in vitro. This approach showed that synovial fluid neutrophils of patients with rheumatoid arthritis have features which indicate that they have been both primed and activated in situ within joints.

Since the discovery that neutrophils isolated from the bloodstream of patients with acute bacterial infections had potentiated oxidative responses ${ }^{18}$ it has become realised that bloodstream neutrophils can be primed in vitro (and presumably in vivo) into a state of enhanced responsiveness after exposure to agents such as chemotactic factors and certain cytokines. ${ }^{37}$ Primed cells thus produce greater amounts of oxidants, are more phagocytic, and have greater cytotoxic activity than control cells. ${ }^{34}$ In view of the fact that some cytokines are detectable in the synovial fluid of patients with inflammatory joint disease, and that incubation of bloodstream neutrophils with isolated synovial fluids induces priming, ${ }^{16}{ }^{17}$ it seems highly probable that neutrophils become primed into this state of enhanced responsiveness within these joints. Our data support this conclusion for the following reasons. Firstly, whereas bloodstream neutrophils could be readily primed in vitro those isolated from synovial fluid of the same patients could not: this suggests that the synovial fluid cells might have been previously exposed to such agents within the joint. Secondly, cells primed in vitro showed enhanced (two to threefold) fMet-Leu-Phe mediated stimulation of luminol dependent chemiluminescence, whereas the response to PMA was only potentiated by $0-20 \%$ (fig 5): neutrophils from $63 \%$ of the synovial fluid samples produced a greater fMet-Leu-Phe stimulated luminol-dependent chemiluminescence than did corresponding bloodstream cells.

Possibly, however, some of the changes in synovial fluid neutrophil responses that we noted may be due to the fact that the cells in synovial effusions are somewhat older than those in the circulation. Furthermore, there may be factors within synovial fluid, distinct from activating and priming agents, which may down-regulate neutrophil responsiveness to the agents that we have tested. Such factors would also be of considerable importance in understanding the complex molecular processes which regulate neutrophil function within inflamed joints. Interestingly, also, the oxidative responses of bloodstream neutrophils from patients with rheumatoid arthritis varied over a greater range ( 50 -fold) than those of normal controls. This observation clearly requires a more extensive study to determine if this is due to inherent functional differences in these patients, increased neutrophil longevity, or whether treatment contributes to this phenomenon. A further investigation in patients with different types of inflammatory conditions may also be appropriate to discover whether this is a more widespread phenomenon associated with inflammation in general.

In addition to developing an enhanced ability to generate reactive oxidants, protein biosynthesis is also activated de novo in primed 
neutrophils. 363839 During this latter process these primed cells develop the ability to generate and secrete some cytokines and colony stimulating factors. ${ }^{4041}$ Thus neutrophils primed in vivo within rheumatoid joints will have an enhanced ability to generate tissue damaging reactive oxidants and may also generate proinflammatory signals, which will aid the recruitment and rate of proliferation of inflammatory cells. Further work is clearly necessary to establish this possibility.

Several independent lines of evidence now suggest that neutrophils within rheumatoid joints have also been activated to secrete reactive oxidants. This evidence comes from experiments measuring surface antigens, ${ }^{12} 13$ oxidative metabolism, ${ }^{16}$ and granule enzyme content. ${ }^{15}$ The data presented here substantiate these proposals of activation in situ based on the following facts. Firstly, all the synovial fluid neutrophils analysed had greatly reduced levels of myeloperoxidase (fig 4): consequently, the responsiveness of these cells to stimulation of luminol dependent chemiluminescence by fMet-Leu-Phe plus cytochalasin B was greatly reduced (fig $3 A$ ) because this response largely requires the activity of an extracellular myeloperoxidase $-\mathrm{H}_{2} \mathrm{O}_{2}$ system. ${ }^{32}$ Secondly, the luminol dependent chemiluminescence response of synovial fluid neutrophils to PMA was lower than that of bloodstream neutrophils (fig 2A): bloodstream neutrophils activated in vitro also had a down-regulated oxidative response upon stimulation by this agent (fig 7). Interestingly, it has also been reported that neutrophils exuded into aseptic skin windows had enhanced fMetLeu-Phe stimulated luminol dependent chemiluminescence but a reduced PMA response. ${ }^{42}$

Therefore, we conclude that synovial fluid neutrophils from patients with rheumatoid arthritis have been both primed and activated in vivo. These cells thus should have increased surface receptor expression (which potentially will enable them to mount a greater oxidative response), but will also have a down-regulated oxidase (as evidenced by the PMA response), which will somewhat reduce their ability to generate oxidants in response to further stimulation. The interplay between these contradictory up- and down-regulatory processes will therefore dictate how the neutrophil population will respond to further stimulation: this population is thus in a dynamic state depending upon the prevailing (and perhaps changing) local conditions within the joint. This phenomenon may help to explain some of our apparently conflicting data (figs 1 and 3 ). Thus where responses of synovial fluid neutrophils are reduced (compared with bloodstream cells) this may indicate that most of the cells have been activated: conversely, when the response of the synovial fluid neutrophils is greater than that of bloodstream cells it may indicate that most of these cells are in the primed state. It is highly likely that the neutrophil population within the inflamed joint is functionally heterogeneous with individual cells in resting, primed, activated, or deactivated states. Further work is necessary to confirm this idea and this will undoubtedly require single cell measurements.
We thank the Arthritis and Rheumatism Council for generous financial support.

1 Curnutte J T, eds. Phagocyte defects $I$ and II. Philadelphia: Saunders. Hematology and Oncology Clinics of North America, 1988: 336

2 Halliwell B, Gutteridge J M C. Free radicals in biology and medicine. Oxford: Clarendon Press, 1985: 346.

3 Babior B M. Oxidants from phagocytes: agents of defence and destruction. Blood 1984; 64: 959-66.

4 Brown $\mathrm{K} \mathrm{A}$. The polymorphonuclear cell in rheumatoid arthritis. Br $\mathcal{J}$ Rheumatol 1988; 27: 150-5.

5 McCord J M. Free radicals and inflammation: protection of synovial fluid by superoxide dismutase. Science 1974; 185: 529-31.

6 Blake D R, Hall N D, Terby D A, Halliwell B, Gutteridge J M C. Protection against superoxide and hydrogen peroxide in synovial fluid from rheumatoid joints. Clin Sci 1981; 61: 483-6.

7 Merry P, Winyard P G, Morris C J, Grootveld M, Blake D R. Oxygen free radicals, inflammation, and synovitis: the current status. Ann Rheum Dis 1989; 48: 864-70.

8 Gale R, Bertouch J V, Bradley J, Roberts-Thomson P J. Direct activation of neutrophil chemiluminescence by rheumatoid sera and synovial fluid. Ann Rheum Dis 1983; 42: $158-62$.

9 Blackburn W D Jr, Koopman W J, Schrohenloher R E, Heck $L W$. Induction of neutrophil enzyme release by rheumatoid factors: evidence for differences based on molecular characteristics. Clin Immunol Immunopathol 1986; 40: characte

10 Andrews F J, Blake D R, Freeman J, et al. Free radicals and perfusion injury in the inflamed joint. In: Swaak A J G, Koster J F, eds. Free radicals and arthritic diseases. Ryswick: Eurage, 1986: 167-178.

11 Merry P, Unsworth J, Lunec J, et al. Exercise induces oxidative damage to synovial fluid IgG. $\mathrm{Br} \mathcal{F}$ Rheumatol 1988; 17 (suppl 2): 121 .

12 Breedveld F C, Lafeber G J M, van den Barselaar T T, van Dissel J T, Leiih P C J. Phagocytosis and intracellular killing of Staphylococcus aureus by polymorphonuclear cells from synovial fluid of patients with rheumatoid arthritis. Arthritis Rheum 1986; 29: 166-73.

13 Emery P, Lopez A F, Burns G F, Vadas M A. Synovial fluid neutrophils of patients with rheumatoid arthritis have membrane antigen changes that reflect activation. Ann membrane antigen changes
Rheum Dis 1988; 47: 34-9.

14 Klebanoff S J, Clark R A. The neutrophil: function and clinical disorders. Amsterdam: North-Holland, 1978: 810 .

15 Edwards S W, Hughes V, Barlow J, Bucknall R. Immunological detection of myeloperoxidase in synovial fluids from patients with rheumatoid arthritis. Biochem $\mathcal{F}$ 1988; 250: 81-5.

16 Bender J G, van Epps D E, Searles R, Williams R C Jr. Altered function of synovial fluid granulocytes in patients with acute inflammatory arthritis: evidence for activation of neutrophils and its mediation by a factor present in synovial fluid. Inflammation 1986; 10: 443-53.

17 Dularay B, Elson C J, Dieppe P A. Enhanced oxidative response of polymorphonuclear leukocytes from synovial rluids of patients with rheumatoid arthritis. Autoimmunity fluids of patients

18 McCall C E, Bass D A, DeChatalet L R, Link A S L Jr, Munn M. In vitro responses of human neutrophils to $\mathbf{N}$ Munn M. In vitro responses of human neutrophils to $\mathrm{N}$ formyl-methionyl-leucyl-phenylalanine: correlation with
effects of acute bacterial infection. F Infect Dis 1979; 140: effects of

19 Steinbeck M J, Roth J A. Neutrophil activation by recombinant cytokines. Rev Infect Dis 1989; 11: 549-68.

20 Moissec $P$. The role of interleukin 1 in the pathogenesis of rheumatoid arthritis. Clin Exp Rheumatol 1987; 5: 305-8.

21 Rafter G W. Interleukin 1 and rheumatoid arthritis. Med Hypotheses 1988; 27: 221-4

22 Yamagata N, Kobayashi K, Kasama T, et al. Multiple cytokine activities and loss of interleukin 2 inhibitor in synovial fluids of patients with rheumatoid arthritis $f$ Synovial fluids of patients with

23 Westacott C I, Whicher J T, Thompson D, Barnes I C, Swan A J, Dieppe P A. Synovial fluid concentration of five different cytokines in rheumatic diseases. $B r \mathcal{F}$ Rheumatol different cytokines in rhe
$1988 ; 17$ (suppl 2): 67 .

24 Hirano T, Matsuda T, Turner M, et al. Excessive production of interleukin $6 / \mathrm{B}$ cell stimulatory factor- 2 in rheumatoid of interleukin 6/B cell stimulatory factor-2 in rhe
arthritis. Eur $\mathcal{F}$ Immunol 1988; 18: 1797-801.

25 McKenna R M, Wilkins J A, Warrington R J. Lymphokine production in rheumatoid arthritis and systemic lupus production in rheumatoid arthritis and systemic

26 Hopkins S J, Meager A. Cytokines in synovial fluid: II. The popkins S J, Meager A. Cytokines in synovial fluid: II. The
presence of tumour necrosis factor and interferon. Clin Exp Immunol 1988; 73: 88-92.

27 Yocum D E, Esparza L, Dubry S, Benjamin J B, Volz R, Scudes $P$. Characteristics of tumour necrosis factor production in rheumatoid arthritis. Cell Immunol 1989; 122: $131-45$.

28 Edwards S W, Say J E, Hart C A. Oxygen-dependent killing of Staphylococcus aureus by human neutrophils. $\mathcal{F}$ Gen Microbiol 1987; 133: 3591-7.

29 Edwards S W. Luminol- and lucigenin-dependent chemiluminescence of neutrophils: role of degranulation. $\mathcal{f}$ Clin Lab Immunol 1987; 22: 35-9.

30 Babior B M, Kipnes R S, Curnutte J T. Biological defense mechanisms: the production by leukocytes of superoxide, a potential bacteriocidal agent. F Clin Invest 1973; 52: 741-4. 
31 Putte J. Peroxidases. In: Bergmeyer H U, ed. Methods in enzymatic analysis. Weinheim: Verlag-Chemie, 1974:

32 Nurcombe H L, Edwards SW. Role of myeloperoxidase in intracellular and extracellular chemiluminescence of neutrophils. Ann Rheum Dis 1989; 48: 56-62.

33 Edwards S W, Hallett M B, Lloyd D, Campbell A K. Decrease in apparent $\mathrm{Km}$ for oxygen after stimulation of respiration of rat polymorphonuclear leukocytes. $F E B S$ respiration of rat poly

34 Edwards S W, Nurcombe H L, Hart C A. Oxidative inactivation of myeloperoxidase released from human neutrophils. Biochem $\mathcal{f}$ 1987; 245: 925-8.

35 Edwards S W, Say J E, Hughes V. Gamma interferon enhances the killing of Staphylococcus aureus by human neutrophils. f Gen Microbiol 1988; 134: 37-42.

36 Edwards S W, Holden C S, Humphreys J M, Hart C A. Granulocyte-macrophage colony-stimulating factor (GMCSF) primes the respiratory burst and stimulates protein biosynthesis in human neutrophils. FEBS Lett 1989; 256: 62-6.

37 Edwards $S$ W. Interactions between bacterial surfaces and phagocyte plasma membranes. Biochem Soc Trans 1989; 17: 460-2.

38 Hughes V, Humphreys J M, Edwards S W. Protein synthesis is activated in primed neutrophils: a possible role in inflammation. Biosci Rep 1987; 7: 881-9.

39 Humphreys J $M$, Hughes V, Edwards S W. Stimulation of protein synthesis in human neutrophils by $\gamma$-interferon. protein synthesis in human neutrophis
Biochem Pharmacol 1989; 38: 1241-6.

40 Lindemann A, Riedel D, Oster W, et al. Granulocyte/macrophage colony-stimulating factor induces interleukin production by human polymorphonuclear neutrophils. f Immunol 1988; 140: 837-9.

41 Lindemann A, Riedel D, Oster W, Ziegler-Heitbrock H W L, Mertlesmann R, Herrmann F. Granulocytemacrophage colony-stimulating factor induces cytokine secretion by human polymorphonuclear leukocytes. F Clin Invest 1989; 83: 1308-12.

42 Briheim G, Coble B, Stendahl O, Dahlgren C. Exudate polymorphonuclear leukocytes isolated from skin chambers are primed for enhanced response to subsequent stimulation with chemoattractant $\mathrm{fMet}-\mathrm{Leu}$-Phe and C3-opsonised yeast particles. Inflammation 1988; 12: 141-52. 